\title{
Pollution levels of DDTs and their spatiotemporal trend from sediment records in the Southern Yellow Sea, China
}

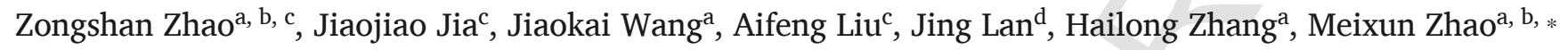 \\ ${ }^{a}$ Key Laboratory of Marine Chemistry Theory and Technology, Ocean University of China, Ministry of Education, China \\ ${ }^{\mathrm{b}}$ Laboratory for Marine Ecology and Environmental Science, Qingdao National Laboratory for Marine Science and Technology, Qingdao 266000, China \\ c CAS Key Laboratory of Bio-based Materials, Qingdao Institute of Biomass Energy and Bioprocess Technology, Chinese Academy of Sciences, Qingdao 266101, China \\ ${ }^{\mathrm{d}}$ College of Chemistry and Pharmaceutical Sciences, Qingdao Agriculture University, Qingdao 266109, China
}

\section{ARTICLE INFO}

\section{Keywords:}

DDTs

Sediment

Pollution levels

Ecological risk

Temporal trend

\begin{abstract}
A B S T R A C T
In order to study the pollution levels and spatiotemporal trend of Dichlorodiphenyltrichloroethanes (DDTs) in the Southern Yellow Sea (SYS), thirty-two surface sediment samples and a sediment core have been analyzed, and our results have been compared with previous reports. DDTs contents in our samples ranged from below detection limit to $5.1 \mathrm{ng} / \mathrm{g}$ dry weight (d.w.), which presented lower ecological risks in the SYS. Surface sediment results show a seaward increasing trend with high values in the northern region of the central basin of the SYS. Our reconstructed core record and historical data from previous reports reveal an increasing trend from 1905 to 1955 but a decline trend since 1985 for DDTs, which is consistent of the production, usage and banning of DDTs in China. The source identification, based on (DDE + DDD) / DDTs, suggested that aged DDTs were the major contributor, though there were some inputs of fresh DDTs from the usage of 1,1-bis(p-Chlorophenyl)-2,2,2-trichloroethanol (dicofol).
\end{abstract}

\section{Introduction}

Dichlorodiphenyltrichloroethanes (DDTs), as a class of semi-volatile persistent organic pollutants (POPs), have been designated as major pollutants of global concern for decades because of their environmental persistence, bioaccumulation, and ecological risks to plants, animals and human (Aigner et al., 1998; Lin et al., 2012b). In China, DDTs were widely used in agriculture as well as in malaria control, and the total production reached 270,000 t from 1960 to 1980 (Qiu et al., 2005). As a result, large amounts of DDTs were exposed to the environment. Due to their serious threats to ecosystems and human health, industrial production of DDTs was banned thereafter in 1983 and the outputs of DDTs declined (Zhang et al., 1999; Li et al., 2015). However, it has been indicated that some fresh DDTs still have been discharged into the environment as a result of export demand and dicofol production (Zhou et al., 2006; Li et al., 2015). As a synthetic com- pound with a high impurity of DDTs, dicofol, 2,2,2-trichloro-1,1-bis(4-chlorophenyl)ethanol, is extensively used for controlling mites, but it is harmful to cotton, fruit trees and vegetables. During dicofol production and usage processes, a high proportion of DDTs has been leaked into the environment (Qiu et al., 2005; Da et al., 2014).

Since organic contaminants in marine sediments can be accumulated over seasonal to decadal timescales, oceans often act as remote monitoring sites of regional distribution and temporal trend of POPs changes (Zhang et al., 1999; Wurl et al., 2006; Zhang et al., 2007; Lohmann et al., 2009; Lin et al., 2012b). The Yellow Sea (YS), one of the China marginal seas, is surrounded by China mainland on the west and the Korean Peninsula on the east (Zhang et al., 2008). Although the Yellow River and Yangtze River do not directly discharge into the YS, large amounts of terrestrial materials from these two rivers have been deposited in the YS due to transports by ocean currents (Huh and Su, 1999; Lim et al., 2007). In addition, the coastal areas of the southern YS (SYS) are densely populated, and aquaculture is highly

\footnotetext{
* Corresponding author at: Key Laboratory of Marine Chemistry Theory and Technology, Ocean University of China, Ministry of Education, China.

Email address: maxzhao@ouc.edu.cn (M. Zhao)
} 
developed (Yin et al., 2015). As a result approximately 7.6\% of contaminants from municipal and industrial activities around the SYS coastal regions have been discharged into the SYS (China's State Oceanic Administration (CSOA), 2007; Ministry of Environmental Protection of the People's Republic of China (MEPPRC), 2007; Duan et al., 2013). Therein, marine sediments from the SYS have been used to determine POPs pollution levels and to evaluate their ecological risks (Shi et al., 2011; Da et al., 2013; Chen et al., 2014).

The contents and distributions of DDTs and their metabolites were often the focus in the early studies in the SYS. It has been noted that they were all presented at lower levels in the SYS, and the effects of human activities in the coastal areas were much greater than in the central SYS basin (Ma et al., 2001). For example, the contents of DDTs in the sediments collected in 2000 from the eastern part of the YS mud area ranged from 0 to $1.29 \mathrm{ng} / \mathrm{g}$ d.w. (Oh et al., 2005). While DDTs were at higher levels (average $7.72 \pm 10.88 \mathrm{ng} / \mathrm{g}$ d.w.) in most sites in Haizhou Bay (Fig. 1) littoral regions of Jiangsu Province. Only one site the level exceeded the guideline for effect range median (ERM) (Long et al., 1998), with surface DDT content up to $62.9 \mathrm{ng} / \mathrm{g}$ d.w. (Liu et al., 2008b). Recently, the major source of DDTs was switched from industrial production into the usage of dicofol after the ban of industrial DDTs (Qiu et al., 2005; Liu et al., 2008b). Though the above limited data have indicated lower ecological risks of DDTs in the SYS, but their current and future risks are still uncertain possibly as a result of the resuspension from sediments caused by cyclonic eddy (Alexander et al., 1991; Bi et al., 2011; Hu et al., 2013), as well as from new inputs (Wong et al., 2005; Yin et al., 2015).

Previous studies have focused on the coastal areas of the SYS spatially, and few sediment records of DDTs were obtained to evaluate the temporal trend. In this study, thirty-two surface sediment samples and a sediment core from the central SYS have been collected for studying pollution levels, spatiotemporal trend and sources of DDTs, especially for the basin areas. Historical data of DDTs in both the coastal and the central SYS have also been compared here to evaluate their ecological risks.

\section{Materials and methods}

\subsection{Chemicals used in the study}

Isotope labeled compounds $\left[{ }^{13} \mathrm{C}_{12}\right]$ o,p'-DDE, $\left[{ }^{13} \mathrm{C}_{12}\right] \mathrm{p}, \mathrm{p}^{\prime}-\mathrm{DDE}$, $\left[{ }^{13} \mathrm{C}_{12}\right]$ o,p'-DDD, $\left[{ }^{13} \mathrm{C}_{12}\right]$ p,p'-DDD, $\left[{ }^{13} \mathrm{C}_{12}\right]$ o,p'-DDT and $\left[{ }^{13} \mathrm{C}_{12}\right]$ p,p'-DDT $(0.5 \mathrm{mg} / \mathrm{L})$ were purchased from Wellington Laboratories, Inc., Canada. Pentachloronitrobenzene (PCNB) was acquired from Cerilliant, Cambridge Isotope Laboratories (Tewksbury, MA), and organochlorine pesticide standard mixture (US-1128), decachlorobiphenyl (PCB 209) and 2,4,5,6-tetrachloro-m-xylene (TCmX) were acquired from Ultra Scientific USA. HPLC grade hexane and dichloromethane as well as $\mathrm{Na}_{2} \mathrm{SO}_{4}$ were purchased from Sigma-Aldrich.

\subsection{Collection of samples}

The YS joins the Bohai Sea in the north and the East China Sea (ECS) in the south. It is separated into the SYS and the Northern Yellow Sea (NYS) by the Shandong Peninsula (Fig. 1). The Yellow River and Yangtze River provide substantial sediment inputs into the SYS, although both do not discharge directly into the SYS (Huh and Su, 1999; Lim et al., 2007). Short sediment cores from thirty-two sites were collected from the coast to the central basin of the SYS during a cruise in April 2012 (Fig. 1), using a stainless steel box-corer onboard the R/V Dong Fang Hong 2 of the Ocean University of China, Qingdao, China. Surface sediments $(0-3 \mathrm{~cm})$ from these sites were used for this study. Core N02 $\left(122.634{ }^{\circ} \mathrm{E}, 36.050{ }^{\circ} \mathrm{N}\right.$, water depth of $\left.64 \mathrm{~m}\right)$ was collected in the mud area of the SYS with a box corer during the cruise in 2011. The core was sectioned at $1 \mathrm{~cm}$ intervals to the depth

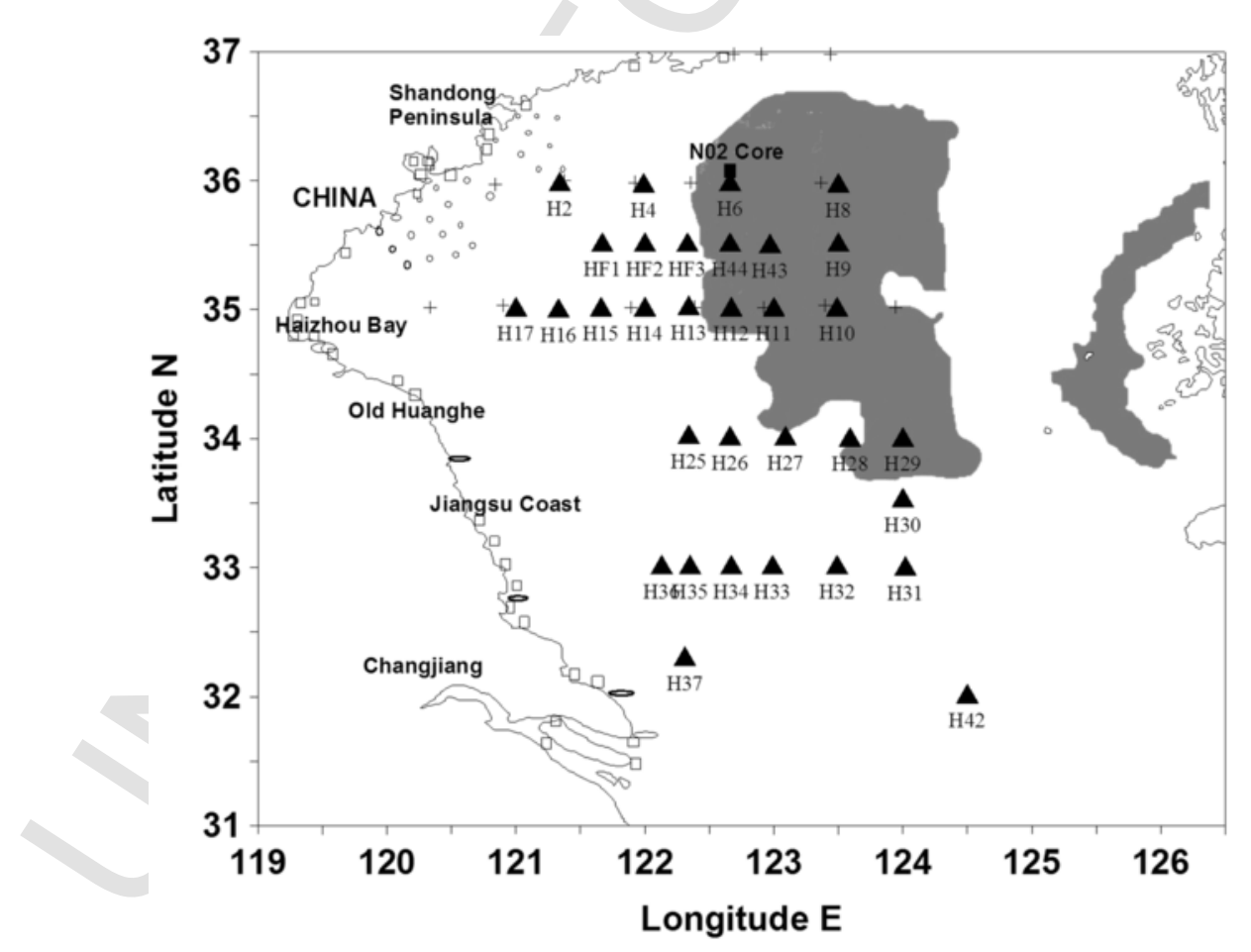

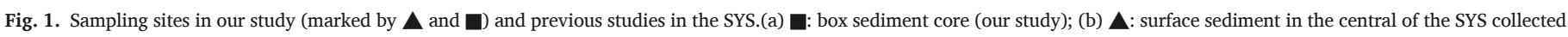

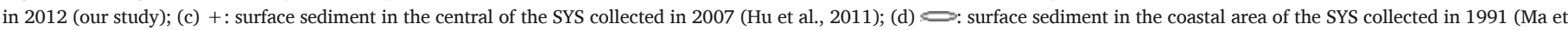

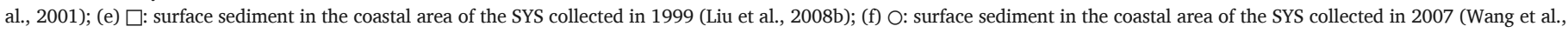
2010). Mud areas are marked in gray. 
of $33 \mathrm{~cm}$ for reconstructing DDT record. All samples were freeze-dried, homogenized, sieved through a stainless steel 75-mesh $(0.5 \mathrm{~mm})$ sieve and stored at $-20{ }^{\circ} \mathrm{C}$ until analysis.

\subsection{Extraction and cleanup}

Sediment samples (10 g) were spiked with surrogates of $50 \mathrm{ng}$ TCmX and PCB209, then suspended with $3 \mathrm{~g}$ anhydrous $\mathrm{Na}_{2} \mathrm{SO}_{4}$ and ultrasonically extracted with $80 \mathrm{~mL}$ hexane/dichloromethane $(\mathrm{v} / \mathrm{v}=1: 1)$ solution for $40 \mathrm{~min}$ and further kept overnight before collecting the extract. Then the sample was extracted again with $80 \mathrm{~mL}$ hexane/dichloromethane $(\mathrm{v} / \mathrm{v}=1: 1)$ solution for another $30 \mathrm{~min}$ and with $20 \mathrm{~mL}$ hexane for $20 \mathrm{~min}$ respectively. Activated copper powder was used for desulphurization. All the extracts were rotary-evaporated to about 1-2 mL and further cleaned with a multilayer composite column consisted of activated silica gel ( $3 \mathrm{~g})$, acid silica gel $(6 \mathrm{~g})$, activated silica gel (1 g) and anhydrous $\mathrm{Na}_{2} \mathrm{SO}_{4}(3 \mathrm{~g})$ from bottom to top. The column was pre-cleaned with $60 \mathrm{~mL}$ dichloromethane/hexane $(1: 5, \mathrm{v} / \mathrm{v})$ before loading each sample and then the extract was eluted in sequence with $30 \mathrm{~mL}$ of hexane and $30 \mathrm{~mL}$ of dichloromethane/hexane (1:9, v/v). The eluate was concentrated with a rotary-evaporator and then concentrated to $0.2 \mathrm{~mL}$ under gentle $\mathrm{N}_{2}$ flow. The internal standard PCNB was added before gas chromatography mass spectrometry (GC-MS) analysis (Doong et al., 2002; Hu et al., 2009).

\subsection{Instrument analysis}

DDTs analysis was performed on an Agilent 6980N gas chromatography-electron capture detection (GC-ECD) system (Agilent Technologies Inc., Palo Alto, USA) equipped with a HP-1 capillary column $(50 \mathrm{~m} \times 0.32 \mathrm{~mm}, 0.17 \mu \mathrm{m})$. Detector temperature was maintained at $300{ }^{\circ} \mathrm{C}$. Splitless injection of $1 \mu \mathrm{L}$ sample was performed with $1 \mathrm{~min}$ solvent delay time, and the injector temperature was set at $250{ }^{\circ} \mathrm{C}$. $\mathrm{N}_{2}$ was used as the carrier gas at a flow rate of $1.0 \mathrm{~mL} / \mathrm{min}$. The oven temperature program started at $80{ }^{\circ} \mathrm{C}$ and held for $2 \mathrm{~min}$, in- creased to $200{ }^{\circ} \mathrm{C}$ at $6{ }^{\circ} \mathrm{C} / \mathrm{min}$, then increased to $250{ }^{\circ} \mathrm{C}$ at $4{ }^{\circ} \mathrm{C} / \mathrm{min}$ and held for $6 \mathrm{~min}$, and finally ramped to $280{ }^{\circ} \mathrm{C}$ at $4{ }^{\circ} \mathrm{C} / \mathrm{min}$ and held for $3 \mathrm{~min}$.

\subsection{Quality assurance and quality control (QA/QC)}

A procedural blank $\left(\mathrm{Na}_{2} \mathrm{SO}_{4}\right)$ and a spiked sample consisting of all reagents were included with each batch of 10 samples to evaluate the possibility of interference and cross-contamination. The recoveries for the surrogate standard, PCB 209 and TCmX, were in the range of $55.8-127.2 \%$. Spiking recoveries of DDTs from the sediments were $45.6 \%-102.7 \%$. Method detection limits of DDTs ranged from 0.20 to $0.90 \mathrm{ng} / \mathrm{g}$ dry weight (d.w.). All the DDTs contents were corrected with the internal standards.

\subsection{Sediment dating}

To obtain ${ }^{210} \mathrm{~Pb}_{\mathrm{ex}}$, the total ${ }^{210} \mathrm{~Pb}$ and the fraction of the total ${ }^{210} \mathrm{~Pb}$ supported by ${ }^{226} \mathrm{Ra}$, were measured for core N02. Gamma spectrometry was employed to measure the activities of ${ }^{210} \mathrm{~Pb}$ and ${ }^{214} \mathrm{~Pb}$ simultaneously on the basis of photon energies at 46.52 and $351.99 \mathrm{keV}$. The detailed calculation equations were described in the reference (Fan et al., 2014). The sedimentation rate is about $0.19 \mathrm{~cm} / \mathrm{yr}$ (Xing et al., 2011) and the time span is $173 \mathrm{yr}$.

\section{Results and discussion}

\subsection{Contents and spatial distribution of DDTs}

Total DDTs contents in surface sediments of the SYS ranged from below detection limits to $5.1 \mathrm{ng} / \mathrm{g}$ d.w., with a mean value of $0.8 \pm 1.0 \mathrm{ng} / \mathrm{g} \mathrm{d}$.w. (Fig. 2). The values were comparable to those in most sediments collected from the Guangdong Daya Bay (0.14-20.27 ng/g d.w.) (Qiu et al., 2002) and the Yangtze Estuary-Hangzhou Bay (0.05-16.00 ng/g d.w.) (Chen et al., 1999), but lower

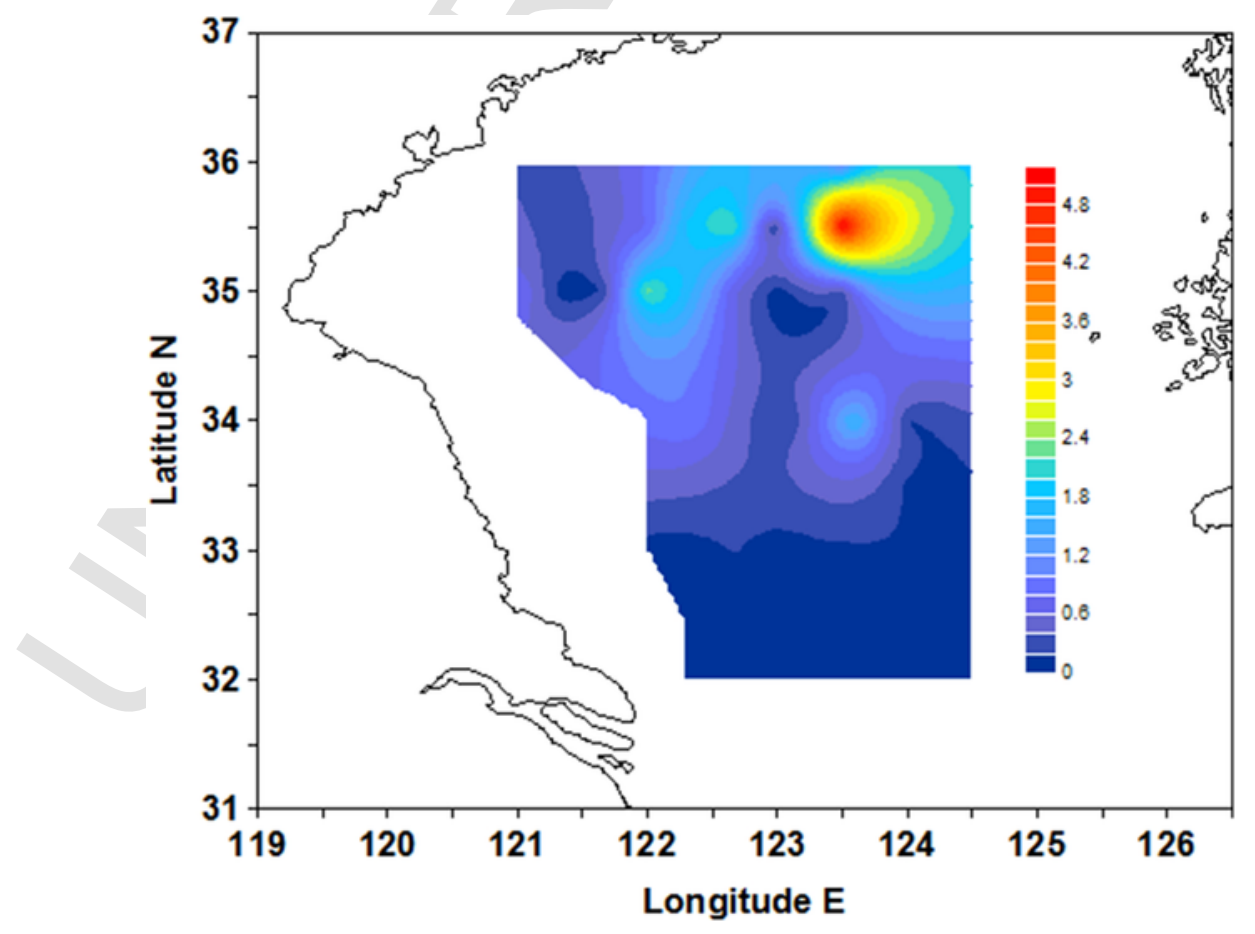

Fig. 2. Spatial distribution of DDTs contents (ng/g d.w.) in surface sediments of the SYS. The gradient distributions were calculated using Kriging by Surfer 8.0. 
than those in sediments adjacent to urbanized areas along the East China Sea (1.2-5.6 ng/g d.w.) (Lin et al., 2012a), the Pearl River Delta area (3.8-31.7 ng/g d.w.) (Mai et al., 2000), the Bohai Sea $(1.36 \pm 0.93 \mathrm{ng} / \mathrm{g}$ d.w.) (Hu et al., 2009) and the mud area in southwest of the Cheju Island (0.80-5.87 ng/g d.w.) (Zhang et al., 2005). Compared with other areas globally, the contents of DDTs in the SYS are also at medium levels, lower than that in Singapore Sea (2.2-11.9 ng/g d.w.) (Wurl and Obbard, 2005) and Vietnam offshore (0.31-274 ng/g d.w.) (Hong et al., 2008), while higher than that in the Gulf of Batabanó and Cuba (0.019-1.27 ng/g d.w.) (Alonso-Hernandez et al., 2014). DDE and DDD are the predominate congeners, with the contents of 0.11-4.88 ng/ g d.w. and ND-0.24 ng/g d.w., respectively. The results were consistent with previous report in the SYS (DDE of 0.05-1.18 and DDD of ND-0.39 ng/g d.w.) (Hu et al., 2011) and in the mud area to southwest of the Cheju Island (DDE of 0.03-0.48 and DDD of 0.03-0.27 ng/g d.w.) (Zhang et al., 2005).

DDTs in the SYS present a seaward-increasing trend characterized with the highest values in the middle and northeast part of the SYS, and this spatial pattern is similar with those revealed by other organic pollutants in the SYS (Hu et al., 2011; Duan et al., 2013). The characteristics can be attributed to the influence of hydrodynamic condition, as it controls the sinking efficiency of fine particles (Hu, 1984) which are prone to adsorb organic matter (Karickhoff et al., 1979). Thus, higher DDT content in the central SYS basin is likely caused by the deposition and accumulation of fine sediments ( $\mathrm{Hu}, 1984)$.

Effects range-low (ERL) and effects range-median (ERM) (Long et al., 1998) have been introduced as interpretive tools to evaluate the ecological risk of DDTs. According to their definition, the ERL and ERM are 1.58 and $46.1 \mathrm{ng} / \mathrm{g}$ d.w. for DDTs respectively. In our study, DDT levels in $78 \%$ of the SYS samples were lower than the ERL value, indicating lower adverse biological effects. The results are comparable to that reported in previous studies in the SYS (0.11-1.4 ng/g d.w.) (Hu et al., 2011). The lower contents of DDTs could be attributed to limited recent input after the usage ban of DDTs since 1983 (Qiu et al., 2005).

\subsection{Temporal trend of DDTs}

To study the historical trends of DDT input to the SYS, sediment core of N02 was collected from the mud area in the central SYS which is a center of organic matter accumulation including organic pollutants, and the results were shown in Fig. 3a. DDTs increased continuously from the core bottom (ca. 1905) to the highest value around 1955, and the value fluctuated from 1955 to 1977 . After that, DDT values presented a decreasing trend. Since 1997, DDTs contents (1.08-1.44 ng/ $\mathrm{g}$ d.w.) were lower than ERL (1.58 ng/g d.w), suggesting lower risks in the central SYS. The reconstructed historical trend of DDTs is broadly consistent with the production and usage history of DDTs in China, characterized by the ban of the industrial DDTs in 1983 (Li et al., 2015). Though the pollution levels of DDTs in sediments decreased since 1977, but the usage and discharge of dicofol still continued and then could lead to fresh input of DDTs (Zhou et al., 2006; Li et al., 2015). Here, its effects on the distributions and risks of DDTs in the SYS cannot be clearly identified due to the mixing of near-surface sediments and the lower sampling resolution of our record.

There were only a few reports on DDTs in the SYS, although central SYS and the coastal areas are both dramatically affected by anthropogenic activities (Lin et al., 2012b; Chen et al., 2014). In this paper, almost all the historical DDTs data of the SYS from this study and previous studies have been compiled and compared to better characterize their historical trend. Fig. 3b shows the comparison of DDTs in surface sediments collected in 2007 and in 2012 (our results). It is found that DDTs contents in all 15 samples (0.11-1.4 ng/g d.w.) collected in 2007 were below the ERL (Hu et al., 2011), slightly lower than those in 2012. The results agreed well with that from the sediment core of N02 (Fig. $3 a)$, indicating that there is no obvious change in DDTs contents in the SYS in recent years. The reason can be attributed to the combined effect of the decreased input of DDTs and the slow deposition of DDTs dissolved in the large body of water. And the low pollution level of DDTs in the surface sediments is likely to continue for a long time.

The published data of DDTs from the coastal areas of the SYS in the past decades have also been collected and compared with our results (Fig. 3c). It is obvious that DDTs contents $(220 \pm 436 \mathrm{ng} / \mathrm{g}$ d.w., 6 samples) in 1991 were much higher than those in $1999(11.2 \pm 17.3 \mathrm{ng} /$ g d.w., 51 samples) and in 2007 (6.0 $\pm 4.7 \mathrm{ng} / \mathrm{g}$ d.w., 29 samples). Among the data collected in 1991, about 33\% of the samples exceeded the ERM and all the samples exceeded the ERL (Ma et al., 2001), suggesting higher biological effects and toxicological risks (Ingersoll et al., 1996). On the other hand for the samples collected in the littoral areas of the SYS in 1999, about $84 \%$ were below ERL. For the samples collected in the SYS in 2007, the contents of DDTs were broadly at similar levels with that collected in 1999. These levels were also similar to those in the surface sediments from the whole area of the SYS and the sediment core in the mud areas, indicating that the
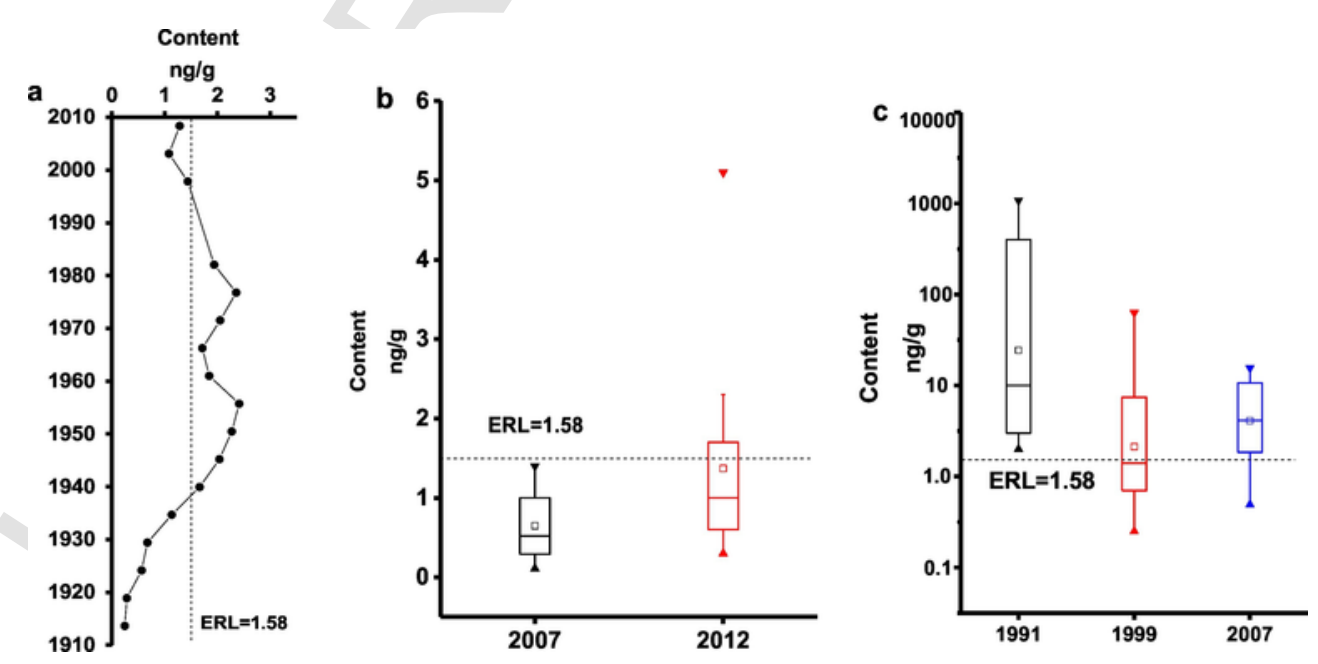

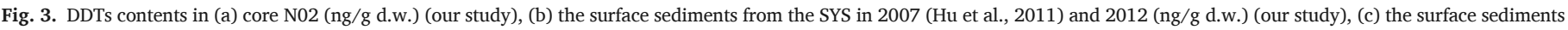
from the coastal area of the SYS (ng/g d.w.) (Ma et al., 2001; Liu et al., 2008b; Wang et al., 2010). The contents of DDTs were calculated using Origin 9.0. 
pollution levels of DDTs in the SYS began to reach a steady state as a result of the continuous deposition of a certain percentage of dissolved DDTs from the water body.

\subsection{Sources of DDTs}

Although the usage of DDTs in agriculture activities was officially banned in 1983 in China, its usage was not terminated until 2000; and dicofol continues to be widely used for controlling mites in China (Qiu et al., 2005; Liu et al., 2008a; Da et al., 2014). Recent studies have indicated that dicofol may be the new source of DDTs in marine environment (Wong et al., 2005; Yin et al., 2015). Here, the indexes of (DDE + DDD) / DDTs (Qiu et al., 2005; Zhang et al., 2011; Da et al., 2014) have been used for studying source changes of DDTs in the SYS. A ratio much lower than 0.5 indicates that there are recent inputs or fresh application of DDTs, whereas a ratio higher than 0.5 indicates aged DDTs (after undergoing microbial degradation) (Zhang et al., 1999; Da et al., 2014).

The ratios of (DDE + DDD) / DDTs from all the studied samples in the central and coastal areas of the SYS are shown in Fig. 4. For the surface sediments from the coastal areas of the SYS, all (DDE + DDD) / DDTs values in 1991 are < 0.5, indicating fresh input domination in sedimental DDTs. After that, about half of (DDE + DDD) / DDTs values (in 1999 and 2007) are > 0.5, suggesting increased contribution of aged DDTs. It must be noted that quite a few number of samples contain fresh DDTs, which indicated their discharge into the coastal areas in recent years, though DDT usages have been banned (Qiu et al., 2005; Li et al., 2015). As for the surface sediment samples collected from the central SYS in 2007 and 2012, the ratio of (DDE + DDD) / DDTs for most samples is higher than 0.5, with only $7 \%$ and $6 \%$ below 0.5 respectively. The same results were also observed in the record of the sediment core N02 (Fig. 4b) with the ratio for all samples much higher than 0.5. Compared with the results in the coastal areas with large numbers of fresh inputs of DDTs (Fig. 4), sedimental DDTs in the central of the SYS should be mainly from the deposition of aged DDTs. This is because before terrestrial matters could be deposited into the sediments of the central SYS, they often undergo long-distance transport and hydrodynamic process of sedimentation and resuspension. The processes often take quite a long time and lead to delayed effect comparing with their deposition in the coastal areas. Additionally, water column biological and biogeochemical processes in the deeper central SYS can also lead to higher ratios of (DDE + DDD) / DDTs in the areas far from the land. In summary, there are some fresh inputs of DDTs into the SYS, but the effect on the pollution level in the central SYS is negligible.

\section{Conclusion}

In this study, surface sediment samples from thirty-two sites and a sediment core from the central SYS have been analyzed to study the pollution levels and the spatiotemporal trends of DDTs. Surface sediment DDTs revealed a seaward increasing trend with high values in the northern region of the central basin of the SYS. Combining our reconstructed record with previous reports of sediment DDTs in this region, a declining trend in recent years and a much lower ecological risks have been revealed. In addition, aged DDTs are proposed to be the major source of surface sediment DDTs, although there are some inputs of free DDTs from recent dicofol usage.

\section{Acknowledgments}

This work was supported by the National Natural Science Foundation of China (21477138, 41521064, 21407086, 41630966), State Key Laboratory of Environmental Chemistry and Ecotoxicology Open Fund (KF2016-12) and the China Postdoctoral Science Foundation (2016M602210). We thank Li Li for technical assistance. We also thank the crew of R/V Dong Fang Hong 2 of the Ocean University of China for the assistance in sediment coring. This is MCTL contribution No. 160.
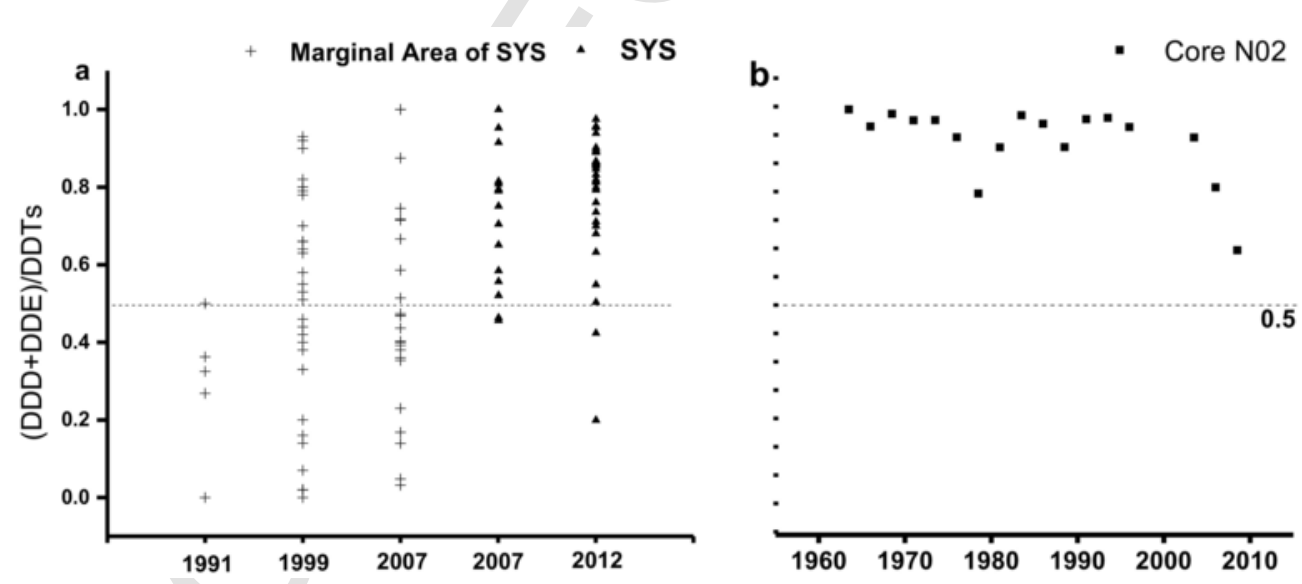

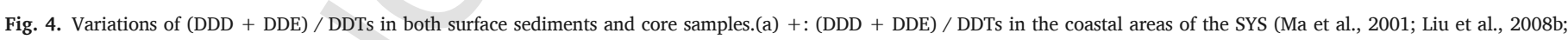

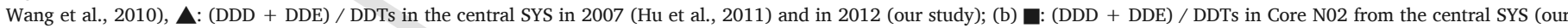
study). The variations were calculated using Origin 9.0. 


\section{References}

Aigner, E.J., Leone, A.D., Falconer, R.L., 1998. Concentrations and enantiomeric ratios of organochlorine pesticides in soils from the U.S. Corn Belt. Environ. Sci. Technol. 32, 1162-1168.

Alexander, C.R., DeMaster, D.J., Nittrouer, C.A., 1991. Sediment accumulation in a modern epicontinental-shelf setting: the Yellow Sea. Mar. Geol. 98, 51-72.

Alonso-Hernandez, C.M., Mesa-Albernas, M., Tolosa, I., 2014. Organochlorine pesticides (OCPs) and polychlorinated biphenyls (PCBs) in sediments from the Gulf of Batabanó, Cuba. Chemosphere 94, 36-41.

Bi, N., Yang, Z., Wang, H., Fan, D., Sun, X., Lei, K., 2011. Seasonal variation of suspended-sediment transport through the southern Bohai Strait. Estuar. Coast. Shelf Sci. 93, 239-247.

Chen, J.f., Ye, X.r., Zhou, H.y., Xia, X.m., Zheng, S.l., 1999. Preliminary study on the marine organic pollution history in Changjiang Estuary-Hangzhou Bay-BHC and DDT stratigraphical records. China Environ. Sci. 19, 206-210.

Chen, J., Chen, L., Liu, D., Zhang, G., 2014. Organochlorine pesticide contamination in marine organisms of Yantai coast, northern Yellow Sea of China. Environ. Monit. Assess. 186, 1561-1568.

China's State Oceanic Administration (CSOA), 2007. Bulletin of China's Marine Environmental Status.

Da, C., Liu, G., Tang, Q., Liu, J., 2013. Distribution, sources, and ecological risks of organochlorine pesticides in surface sediments from the Yellow River Estuary, China. Environ. Sci. Processes Impacts 15, 2288-2296.

Da, C., Liu, G., Yuan, Z., 2014. Analysis of HCHs and DDTs in a sediment core from the Old Yellow River Estuary, China. Ecotoxicol. Environ. Saf. 100, 171-177.

Doong, R.-A., Sun, Y.-C., Liao, P.-L., Peng, C.-K., Wu, S.-C., 2002. Distribution and fate of organochlorine pesticide residues in sediments from the selected rivers in Taiwan. Chemosphere 48, 237-246.

Duan, X., Li, Y., Li, X., Zhang, D., Li, M., 2013. Polychlorinated biphenyls in sediments of the Yellow Sea: distribution, source identification and flux estimation. Mar. Pollut. Bull. 76, 283-290.

Fan, Y., Huh, C.-A., Lan, J., Zhao, M., Zhao, Z., Li, G., Sun, J., Jiang, G., 2014. Major sources of $\mathrm{MeO} / \mathrm{OH}-\mathrm{BDEs}$ in the East China Sea elucidated from their records and phytoplankton biomarkers. Environ. Pollut. 192, 1-8.

Hong, S.H., Yim, U.H., Shim, W.J., Oh, J.R., Viet, P.H., Park, P.S., 2008. Persistent organochlorine residues in estuarine and marine sediments from Ha Long Bay, Hai Phong Bay, and Ba Lat Estuary, Vietnam. Chemosphere 72, 1193-1202.

Hu, D., 1984. Upwelling and sedimentation dynamics. I. The role of upwelling in sedimentation in the Huanghai Sea and East China Sea-a description of general features. Chin. J. Oceanol. Limnol. 2, 13-19.

Hu, L., Zhang, G., Zheng, B., Qin, Y., Lin, T., Guo, Z., 2009. Occurrence and distribution of organochlorine pesticides (OCPs) in surface sediments of the Bohai Sea, China. Chemosphere 77, 663-672.

Hu, L.M., Lin, T., Shi, X.F., Yang, Z.S., Wang, H.J., Zhang, G., Guo, Z.G., 2011. The role of shelf mud depositional process and large river inputs on the fate of organochlorine pesticides in sediments of the Yellow and East China seas. Geophys. Res. Lett. 38, 246-258.

Hu, L., Shi, X., Guo, Z., Wang, H., Yang, Z., 2013. Sources, dispersal and preservation of sedimentary organic matter in the Yellow Sea: the importance of depositional hydrodynamic forcing. Mar. Geol. 335, 52-63.

Huh, C.-A., Su, C.-C., 1999. Sedimentation dynamics in the East China Sea elucidated from ${ }^{210} \mathrm{~Pb},{ }^{137} \mathrm{Cs}$ and ${ }^{239,240} \mathrm{Pu}$. Mar. Geol. 160, 183-196.

Ingersoll, C.G., Haverland, P.S., Brunson, E.L., Canfield, T.J., James Dwyer, F., Henke, C.E., Kemble, N.E., Mount, D.R., Fox, R.G., 1996. Calculation and evaluation of sediment effect concentrations for the amphipod Hyalella azteca and the midge Chironomus riparius. J. Great Lakes Res. 22, 602-623.

Karickhoff, S.W., Brown, D.S., Scott, T.A., 1979. Sorption of hydrophobic pollutants on natural sediments. Water Res. 13, 241-248.

Li, J., Li, F., Liu, Q., 2015. Sources, concentrations and risk factors of organochlorine pesticides in soil, water and sediment in the Yellow River estuary. Mar. Pollut. Bull. 100, 516-522.

Lim, D.I., Choi, J.Y., Jung, H.S., Rho, K.C., Ahn, K.S., 2007. Recent sediment accumulation and origin of shelf mud deposits in the Yellow and East China Seas. Prog. Oceanogr. 73, 145-159.

Lin, T., Hu, L., Shi, X., Li, Y., Guo, Z., Zhang, G., 2012. Distribution and sources of organochlorine pesticides in sediments of the coastal East China Sea. Mar. Pollut. Bull. 64, 1549-1555

Lin, T., Li, J., Xu, Y., Liu, X., Luo, C., Cheng, H., Chen, Y., Zhang, G., 2012. Organochlorine pesticides in seawater and the surrounding atmosphere of the marginal seas of China: spatial distribution, sources and air-water exchange. Sci. Total Environ. 435-436, 244-252.

Liu, M., Cheng, S., Ou, D., Yang, Y., Liu, H., Hou, L., Gao, L., Xu, S., 2008. Organochlorine pesticides in surface sediments and suspended particulate matters from the Yangtze estuary, China. Environ. Pollut. 156, 168-173.

Liu, W., Chen, J., Hu, J., Ling, X., Tao, S., 2008. Multi-residues of organic pollutants in surface sediments from littoral areas of the Yellow Sea, China. Mar. Pollut. Bull. 56, 1091-1103.

Lohmann, R., Gioia, R., Jones, K.C., Nizzetto, L., Temme, C., Xie, Z., Schulz-Bull, D., Hand, I., Morgan, E., Jantunen, L., 2009. Organochlorine pesticides and PAHs in the surface water and atmosphere of the North Atlantic and Arctic Ocean. Environ. Sci. Technol. 43, 5633-5639.

Long, E.R., Field, L.J., MacDonald, D.D., 1998. Predicting toxicity in marine sediments with numerical sediment quality guidelines. Environ. Toxicol. Chem. 17, 714-727.

Ma, M., Feng, Z., Guan, C., Ma, Y., Xu, H., Li, H., 2001. DDT, PAH and PCB in sediments from the intertidal zone of the Bohai Sea and the Yellow Sea. Mar. Pollut. Bull. 42, 132-136.

Mai, B.x., Lin, Z., Zhang, G., Sheng, G.y., Min, Y.s., Fu, J.m., 2000. Organic contaminant in surface sediments from rivers of the Pearl River Delta and Estuary-the distributions and characteristics of PAHs and organochlorine pesticides. Acta Sci. Circumst. 20, 192-197.

Ministry of Environmental Protection of the People's Republic of China (MEPPRC), 2007 China Offshore Marine Environment Quality Bulletin.

Oh, J.R., Choi, H.K., Hong, S.H., Yim, U.H., Shim, W.J., Kannan, N., 2005. A preliminary report of persistent organochlorine pollutants in the Yellow Sea. Mar. Pollut. Bull. 50, 217-222.

Qiu, Y.w., Zhou, J.l., Maskaoui, K., Yan, W., Hong, H.s., Wang, Z.d., 2002. Study on polychlorinated biphenyl congeners and organochlorine insecticides in Daya Bay. Mar. Environ. Sci. 21, 46-51.

Qiu, X.H., Zhu, T., Yao, B., Hu, J., Hu, S., 2005. Contribution of dicofol to the current DDT pollution in China. Environ. Sci. Technol. 39, 4385-4390.

Shi, S., Huang, Y., Zhang, L., Zhang, X., Zhou, L., Zhang, T., Dong, L., 2011. Organochlorine pesticides in muscle of wild seabass and Chinese prawn from the Bohai Sea and Yellow Sea, China. Bull. Environ. Contam. Toxicol. 87, 366.

Wang, J.T., Tan, L.J., Zhang, W.H., Lian, Z.R., 2010. Concentrations and distribution characteristic of PAHs, PCBs and OCPs in the surface sediments of Qingtao coastal area. Environ. Sci. 31, 2713-2722.

Wong, M.H., Leung, A.O.W., Chan, J.K.Y., Choi, M.P.K., 2005. A review on the usage of POP pesticides in China, with emphasis on DDT loadings in human milk. Chemosphere $60,740-752$.

Wurl, O., Obbard, J.P., 2005. Organochlorine pesticides, polychlorinated biphenyls and polybrominated diphenyl ethers in Singapore's coastal marine sediments. Chemosphere 58, 925-933.

Wurl, O., Potter, J.R., Obbard, J.P., Durville, C., 2006. Persistent organic pollutants in the equatorial atmosphere over the open Indian Ocean. Environ. Sci. Technol. 40, 1454-1461.

Xing, L., Tao, S., Zhang, H., Liu, Y., Yu, Z., Zhao, M., 2011. Distributions and origins of lipid biomarkers in surface sediments from the southern Yellow Sea. Appl. Geochem. 26, 1584-1593.

Yin, G., Asplund, L., Qiu, Y., Zhou, Y., Wang, H., Yao, Z., Jiang, J., Bergman, , 2015. Chlorinated and brominated organic pollutants in shellfish from the Yellow Sea and East China Sea. Environ. Sci. Pollut. Res. Int. 22, 1713-1722.

Zhang, G., Min, Y.S., Mai, B.X., Sheng, G.Y., Fu, J.M., Wang, Z.S., 1999. Time trend of BHCs and DDTs in a sedimentary core in Macao Estuary, Southern China. Mar. Pollut. Bull. 39, 326-330.

Zhang, Z.y., Guo, Z.g., Zhang, G., Li, J., Chi, J.s., 2005. Distribution of organochlorine pesticide in the surface sediments of the mud areas in the East China Sea. China Environ. Sci. 25, 724-728.

Zhang, G., Li, J., Cheng, H., Li, X., Xu, W., Jones, K.C., 2007. Distribution of organochlorine pesticides in the northern South China Sea: implications for land outflow and air-sea exchange. Environ. Sci. Technol. 41, 3884-3890.

Zhang, S.W., Wang, Q.Y., Lü, Y., Cui, H., Yuan, Y.L., 2008. Observation of the seasonal evolution of the Yellow Sea Cold Water Mass in 1996-1998. Cont. Shelf Res. 28, 442-457.

Zhang, J., Qi, S., Xing, X., Tan, L., Gong, X., Zhang, Y., Zhang, J., 2011. Organochlorine pesticides (OCPs) in soils and sediments, southeast China: a case study in Xinghua Bay. Mar. Pollut. Bull. 62, 1270-1275.

Zhou, R., Zhu, L., Yang, K., Chen, Y., 2006. Distribution of organochlorine pesticides in surface water and sediments from Qiantang River, East China. J. Hazard. Mater. 137, $68-75$. 